\title{
Flow separation patterns in a 60-degree junction
}

\author{
K. Alhussan \\ Space Research Institute, King Abdulaziz City for Science and \\ Technology, Saudi Arabia
}

\begin{abstract}
The work to be presented herein is a theoretical and numerical analysis of the complex fluid mechanism that occurs inside a $60^{\circ}$-junction shape specifically with regard to the boundary layer separation, vortex shedding and generation of wake. The boundary layer separates from the surface, forms a free shear layer and is highly unstable. This shear layer will eventually roll into a discrete vortex and detach from the surface. A periodic flow motion will develop in the wake as a result of boundary layer vortices being shed from the solid boundary. The periodic nature of the vortex shedding phenomenon can sometimes lead to unwanted structural vibrations, especially when the shedding frequency matches one of the resonant frequencies of the structure.

A number of important conclusions follow from the current research. First, the study of the actual flow configuration inside $60^{\circ}$-junction shape offers some insight into the complex flow phenomena. Second, the characteristics of the boundary layer separation, the vortex and wake change considerably with the divergent angle. This research shows that downstream, away from the junction, the boundary layer reattaches and normal flow occurs, i.e. the effect of the boundary layer separation is only local. Nevertheless fluid downstream of the junction will have lost energy. The results show that downstream, away from the junction, the boundary layer reattaches and normal flow occurs, i.e. the effect of the boundary layer separation is only local. Nevertheless fluid downstream of the junction will have lost energy. This research shows that when the velocity ratio is equal to unity the circulation and the vortex shedding phenomenon are maximized.
\end{abstract}

Keywords: flow separation, vortex flow, internal flow, boundary layer, numerical analysis, CFD. 


\section{Introduction}

Flow separation from the surface of a solid boundaries, and the determination of global changes in the flow field that develop as a result of the flow separation, are the most difficult and fundamental problems of fluid dynamics. Gases and water have extremely small viscosity and, therefore, most practical flows are characterized by very large values of the Reynolds number; both theory and experiment show that increasing Reynolds number almost invariably results in flow separation [1-3].

The difference between a separated flow and its theoretical unseparated flow concerns not only the form of trajectories of fluid particles, but also the value of aerodynamic forces acting on the body. For example, for blunt bodies in an incompressible flow, it is known from experimental studies that the drag force is not equal to zero. It does not approach zero as the Reynolds number becomes large. One of the most famous results of the inviscid flow theory is d'Alembert's paradox which says that solid bodies experience zero drag in incompressible flow. It is well known that this contradiction is associated with the assumption of a fully attached form of the flow; this case never happens in reality.

Separation imposes a considerable limitation and lead to a significant degradation of total performance. For example, separation is usually accompanied by a loss of the lift force and sharp increase of the drag at the reattachment region. The first attempts to describe flow separation past blunt bodies are due to Helmholtz (1868) and Kirchhoff (1869) in the analysis of the classical theory of inviscid fluid flows, but there was no adequate explanation as to why separation occurs. Prandtl (1904) was the first to recognize the physical cause of separation at high Reynolds numbers as being associated with the separation of boundary layers that must form on all solid surfaces [1-5].

In accordance with the Prandtl's theory, a high Reynolds number flow past a rigid body has to be subdivided into two characteristic regions. The main part of the flow field may be treated as inviscid. However, for all Reynolds numbers, no matter how large, there always exists a thin region near the wall where the flow is predominantly viscous. Prandtl termed this region the boundary layer, and suggested that it is because of the specific behaviour of this layer that flow separation takes place. Flow development in the boundary layer depends on the pressure distribution along the wall. If the pressure gradient is favourable then the boundary layer remains well attached to the wall. However with adverse pressure gradient the boundary layer tends to separate from the body surface. The reason for separation was explained by Prandtl in the following way. Since the velocity in the boundary layer drops towards the wall, the kinetic energy of fluid particles inside the boundary layer appears to be less than that at the outer edge of the boundary layer, in fact the closer a fluid particle is to the wall the smaller its kinetic energy. This means that while the pressure rise in the outer flow may be quite significant, the fluid particles inside the boundary layer may not be able to get over it. Even a small increase of pressure may cause the fluid particles near the wall to stop and then turn back to form a recirculating flow region, which is the characteristic of separated flows [2-6]. 
The work to be presented herein is a Computational Fluid Dynamics investigation of the complex fluid mechanisms that occur inside $60^{\circ}$-junction shape, specifically with regard to the boundary layer separation. This research shows a numerical simulation of mapping the flow inside a $60^{\circ}$-junction shape flow. The results show that downstream, away from the junction, the boundary layer reattaches and normal flow occurs i.e. the effect of the boundary layer separation is only local. This research shows that when the velocity ratio is equal to unity the circulation and the vortex shedding phenomenon are maximized.

A CFD analysis enables one to understand the complex flow structure inside this confined region. Through this computational analysis, a better interpretation and understanding of the physical phenomenon of the boundary layer separation that occurs in an internal flow; the $60^{\circ}$-junction shape, can be achieved. Figure 1 shows schematic view of a $60^{\circ}$-junction with two dimensional unstructured mesh.

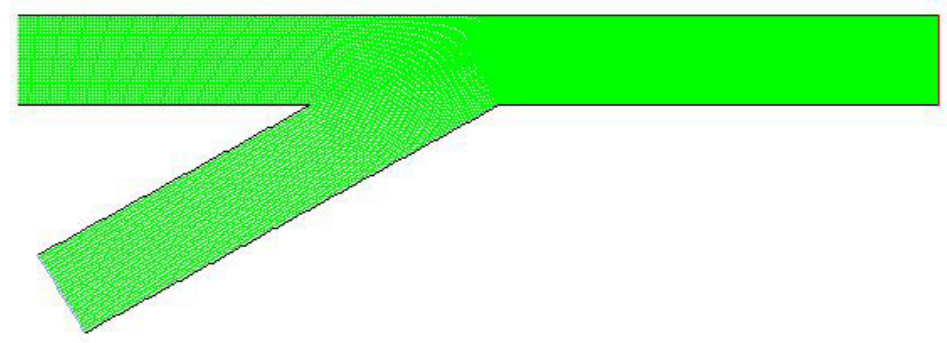

Figure 1: $\quad$ Schematic view of a $60^{\circ}$-junction showing a 2-D unstructured mesh.

\section{Theoretical and numerical analysis}

The governing equations are a set of coupled nonlinear, partial differential equations. In order to formulate or approximate a valid solution for these equations they must be solved using computational fluid dynamics techniques. To solve the equations numerically they must be discretized. That is, the continuous control volume equations must be applied to each discrete control volume that is formed by the computational grid. The integral equations are replaced with a set of linear algebraic equations solved at a discrete set of points.

Fluent is used in this current research to model the flow characteristics inside the two dimensional region. It should be possible to model the flow separation for internal flow inside a $60^{\circ}$-junction shape using the CFD analysis [7-14]. A numerical analysis must start with breaking the computational domain into discrete sub-domains, which is the grid generation process. A grid must be 
provided in terms of the spatial coordinates of grid nodes distributed throughout the computational domain. At each node in the domain, the numerical analysis will determine values for all dependent variables such as pressure and velocity components. Creating the grid is the first step in calculating a flow. Twodimensional Navier Stokes equations are solved using fully implicit scheme with $\mathrm{K}$-epsilon turbulence model. The grid is refined near the solid boundary walls in order to model the large gradient.

\section{Results and discussion}

The work to be presented herein is a theoretical and numerical analysis of the complex fluid mechanism that occurs inside a $60^{\circ}$-junction shape specifically with regard to the boundary layer separation, vortex shedding and generation of wake.

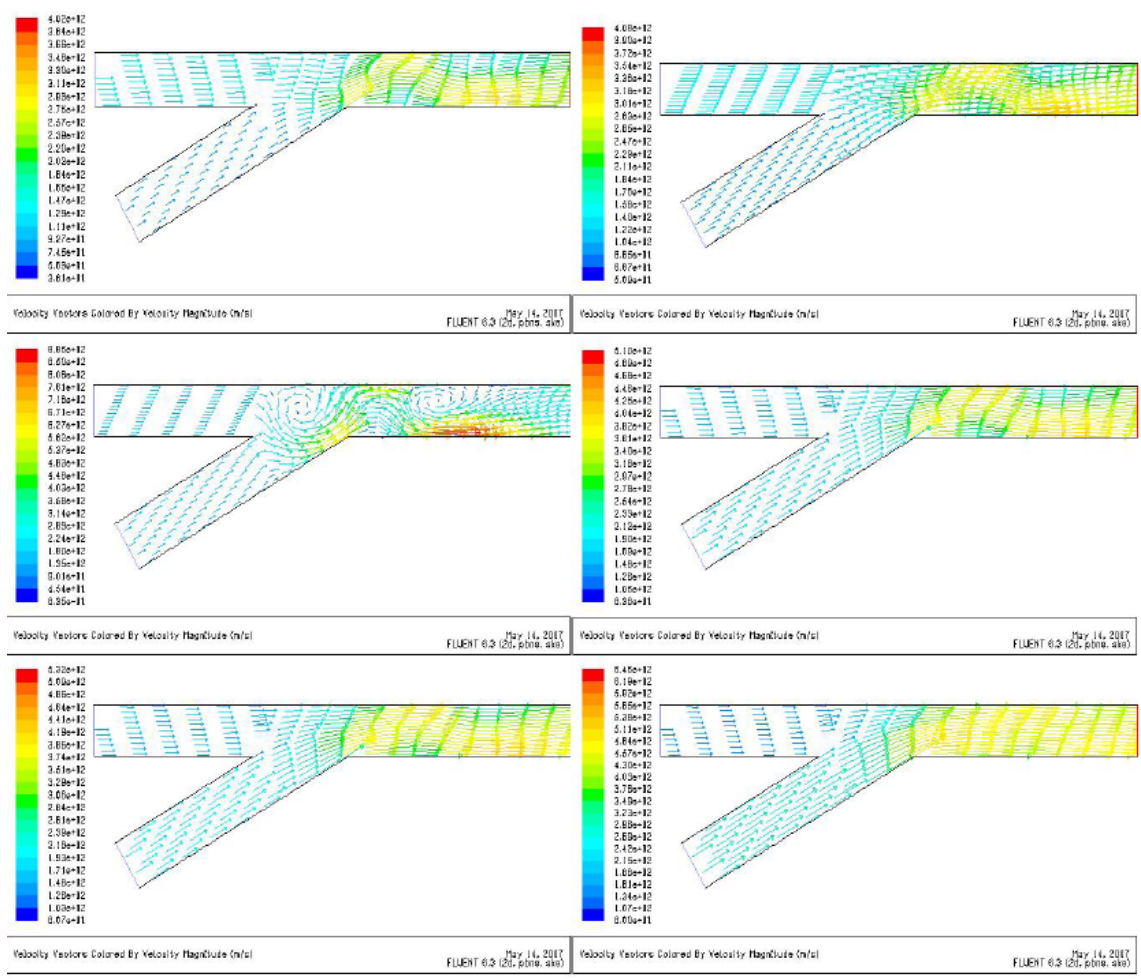

Figure 2: Velocity vectors for flow inside $60^{\circ}$-junction from top left to bottom right the velocity ratio is $0.5,0.67,1,1.16,1.3$, and 2 .

Figure 2 shows velocity vectors for internal flow of a $60^{\circ}$-junction shape. From top left to bottom right the velocity ratio is $0.5,0.67,1,1.16,1.3$, and 2 , respectively. The velocity ratio is defined as the velocity of the branched inlet to 
the velocity of the main (straight) inlet. One can see in this figure that the circulation, wake generation and the vortex shedding phenomenon are evident when the velocity ratio is equal to 1 . This figure shows that downstream, away from the junction, the boundary layer reattaches and normal flow occurs i.e. the effect of the boundary layer separation is only local. Nevertheless fluid downstream of the junction will have lost energy. The boundary layer separates from the surface forms a free shear layer and is highly unstable. This shear layer will eventually roll into a discrete vortex and separate from the surface. A periodic flow motion will develop in the wake as a result of boundary layer vortices being shed from the solid boundary. The periodic nature of the vortex shedding phenomenon can sometimes lead to unwanted structural vibrations, especially when the shedding frequency matches one of the resonant frequencies of the structure.

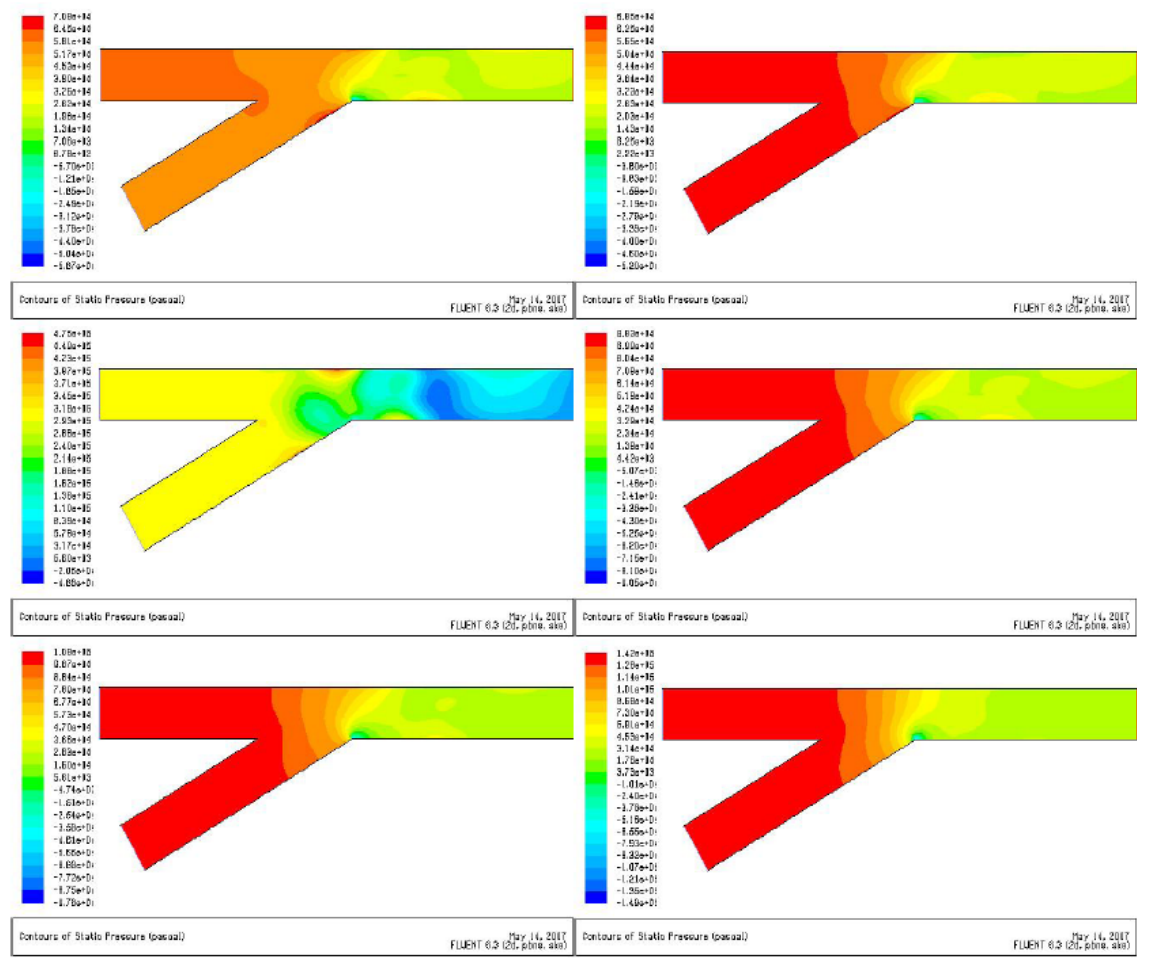

Figure 3: Contour plots of static pressure for flow inside $60^{\circ}$-junction from top left to bottom right the velocity ratio is $0.5,0.67,1,1.16,1.3$, and 2 .

Figure 3 shows contour plots of static pressure for internal flow of a $60^{\circ}$ junction shape. From top left to bottom right the velocity ratio is $0.5,0.67,1$, $1.16,1.3$, and 2 , respectively. One can see in this figure that the circulation is evident when the velocity ratio is equal to 1 . The results show that downstream, 
away from the junction, the boundary layer reattaches and normal flow occurs i.e. the effect of the boundary layer separation is only local. Nevertheless fluid downstream of the junction will have lost energy.

\section{Concluding remarks}

The work to be presented herein is a Computational Fluid Dynamics investigation of the complex fluid mechanisms that occur inside $60^{\circ}$-junction shape, specifically with regard to the boundary layer separation, vortex shedding and generation of wake.

A number of important conclusions follow from the current research. First, study of the actual flow configuration inside the $60^{\circ}$-junction shape offers some insight into the complex flow phenomena. Second, downstream, away from the junction, the boundary layer reattaches and normal flow occurs i.e. the effect of the boundary layer separation is only local. This research showed that when the velocity ratio is equal to unity the circulation and the vortex shedding phenomenon are maximized.

\section{Acknowledgement}

The author gratefully acknowledges sponsorship of this research from the Space Research Institute of the King Abdulaziz City for Science and Technology.

\section{References}

[1] Nesteruk I., "Can Shapes with Negative Pressure Gradients Prevent Cavitation", Proceedings of FEDSM'03,4 ${ }^{\text {Th }}$ ASME JSME Joint Fluids Engineering Conference, Honolulu, USA, July 6-11, 2003, No. FEDSM2003-45323.

[2] Nesteruk I., "Experimental Investigation of Axisymmetric Bodies with Negative Pressure Gradients", Aeronautical Journ., v. 104: 439-443, 2000.

[3] Takahashi S., Washio S., Uemura K. and Okazaki A., "Experimental study on cavitation starting at and flow characteristics close to the point of separation", Fifth International Symposium on Cavitation (Cav2003), Osaka, Japan, No. Cav03-OS-3-003, pp. 1-8, 2003.

[4] Sahu, J. \& Nietubicz, C.'Navier-Stockes Computations of Projectile Base Flow with and without Mass Injection: AIAA Journal, Vol. 23, No. 9, pp.1348-1355, 1985.

[5] Bourdon, C. \& Dutton, J. " Visualization of a Central Bleed Jet in an Axisymmetric Compressible Flow" Physics of Fluids, Vol. 15, No. 2 pp.499-510, 2003.

[6] Perkins, S. C.\& Dillenius, M. “ Supersonic Submunitions Aerodynamics during Dispense" Journal of Spacecraft and Rockets, Vol. 28, No.3 pp.276283, 1991 
[7] Lee, Y., Kim, H. \& Raghunathan, S. "A Study of Base Drag Optimization using Mass Bleed" $15^{\text {th }}$ Australian Fluid Mechanics Conference, Sydney, Australia, 2004.

[8] Lee, Y., Chang, S.Lee, J. \& Kim, H.: A Study of the Aerodynamic Drag of a Blunt Body with an Air Bag" the $6^{\text {th }}$ KSME-JSME Thermal and Fluids Engineering Conference, Jeju City, Korea, 2005.

[9] Alhussan, K., " Computational Analysis of High Speed Flow over a Double-Wedge for Air as Working Fluid", Proceedings of FEDSM2005 ASME Fluids Engineering Division Summer Meeting and Exhibition FEDSM2005-77441 June 19-23, 2005, Houston, TX, USA.

[10] Alhussan, K.," Study the Structure of Three Dimensional Oblique Shock Waves over conical rotor-Vane surfaces", Proceedings of FEDSM2005 ASME Fluids Engineering Division Summer Meeting and Exhibition FEDSM2005-77440 June 19-23, 2005, Houston, TX, USA.

[11] Alhussan, K. ," Oblique Shock Waves Interaction in a Non-Steady Three Dimensional Rotating Flow", Proceedings of FEDSM2005 ASME Fluids Engineering Division Summer Meeting and Exhibition FEDSM2005-77442 June 19-23, 2005, Houston, TX, USA.

[12] Alhussan, K. "Application of Computational Fluid Dynamics in Discontinuous Unsteady Flow with Large Amplitude Changes; The shock Tube Problem" IASME Transaction Issue 1 Volume 2, pp 98-104, January 2005.

[13] Alhussan, K. "Supersonic Flow over Blunt Body with a Decelerator" IASME Transaction ssue 3 Volume 1, pp 8-104, August 2005.

[14] Alhussan, K. " The effect of the boundary layer separation in Y-junction shape" proceeding of the $5^{\text {th }}$ joint ASME/JSME FEDSM2007, Fluid Engineering conference, paper number FEDSM2007-37461, San Diego, CA, USA, July 30-August 2, 2007. 